\title{
Investigation of Mode Filtering as a Preprocessing Method for Shallow-Water Acoustic Communications
}

\author{
Andrey K. Morozov, James C. Preisig, and Joseph C. Papp
}

\begin{abstract}
Acoustical array data from the 2006 Shallow Water Experiment (SW06) was analyzed to show the feasibility of broadband mode decomposition as a preprocessing method to reduce the effective channel delay spread and concentrate received signal energy in a small number of independent channels. The data were collected by a vertical array, which spans the water column from 12-m depth to the bottom in shallow water $80 \mathrm{~m}$ in depth. Binary $m$-sequence data were used to phase-shift-keyed (PSK) modulate signals with different carrier frequencies. No error correction coding was used. The received signals were processed by a system that does not use training or pilot signals. Signals received both during periods of ordinary internal wave activity and during a period with unusually strong internal wave solitons were processed and analyzed. Different broadband mode-filtering methods were analyzed and tested. Broadband mode filtering decomposed the received signal into a number of independent signals with a reduced delay spread. The analysis of signals from the output of mode filters shows that even a simple demodulator can achieve a low bit error rate (BER) at a distance $19.2 \mathrm{~km}$.
\end{abstract}

Index Terms-Signal processing, underwater acoustic arrays, underwater acoustic communications.

\section{INTRODUCTION}

A NUMBER of papers have been dedicated to shallowwater acoustic (SWA) mode problems such as signal mode decomposition and mode filtering and their applications [1]-[14]. Specifically, acoustic normal-mode filtering and mode fluctuations in shallow-water experiments were considered in [10]. The problems in undersized sparse vertical arrays were investigated in [8]. The comparison of different approaches to mode coefficients estimation was conducted in [5], [11], and [12]. In accordance with referenced sources, pseudoinverse transformation (PI) is a very reasonable method for mode coefficient estimation when the signal-to-noise ratio (SNR) is much larger than one. Results for mode fluctuations in the 2006 Shallow Water Experiment (SW06) were first considered by Duda et al. [13], and Collis [15]. The efficiency of mode filtering as a preprocessing method for shallow-water data

Manuscript received January 14, 2009; revised October 14, 2009; accepted November 12, 2009. Date of publication August 26, 2010; date of current version November 30, 2010. This work was supported by the U.S. Office of Naval Research (ONR).

Associate Editor: J. F. Lynch.

A. K. Morozow and J. C. Preisig are with the Department of Applied Ocean Physics and Engineering, Woods Hole Oceanographic Institution, Woods Hole, MA 02543 USA (e-mail: amorozov@ whoi.edu).

J. C. Papp was with the Department of Applied Ocean Physics and Engineering, Woods Hole Oceanographic Institution, Woods Hole, MA 02543 USA. $\mathrm{He}$ is now with the Lincoln Laboratory, Massachusetts Institute of Technology (MIT), Lexington, MA 02421-6426 USA.

Color versions of one or more of the figures in this paper are available online at http://ieeexplore.ieee.org.

Digital Object Identifier 10.1109/JOE.2010.2045444 communications was demonstrated in [16], and was discussed at the 2008 ASA Acoustics Meeting, Paris, France [17].

In this paper, mode filtering is considered as a natural preprocessing spatial-temporal filter to shorten delay spread and decrease the amplitude fluctuations in the channel.

Normal-mode propagation is a natural property of low-frequency sound physics in shallow water, limited in a vertical dimension by sea surface and deep bottom layers. A discrete number of normal modes propagate in shallow water, each with a different group velocity. Although there is modal group velocity dispersion within each mode, each mode waveform has a smaller delay spread and level of fading than the entire multimodal signal. The normal-mode decomposition can essentially increase the performance of quasi-coherent underwater acoustic communications systems to adapt the system rapidly enough to keep up with environmental fluctuations. In addition, array processing will increase the SNR, which can be important in cases where the received SNR is low. This energy limitation is very relevant for communication links over ranges longer than $10 \mathrm{~km}$ and for communication with autonomous and mobile platforms such as autonomous underwater vehicles (AUVs).

The adaptation rate of linear or nonlinear equalizer is limited by the number of degrees of freedom that must be adjusted, specifically by the number of taps in the channel impulse response or equalizer filter. In the cases where the receiver has access to an array with enough sensors and aperture to resolve the propagating modes (e.g., a fixed base station that receives signals from AUVs), wideband modal filtering as an initial processing stage can reduce the number of degrees of freedom (e.g., reduce the effective delay spread of the channel impulse response) that need to be adjusted in later adaptive stages, and provides a method of coherently combining all of the received propagating energy in the water column. The modal preprocessing can reduce the required degrees of freedom in a subsequent adaptive equalizer, because the number of propagating modes and thus the number of modes containing the significant energy is often less than the number of array elements. Therefore, a problem of working on the outputs of $N$ hydrophones can be reduced to the problem of operating on the wideband-modal time series of $M$ modes where $M<N$. The ability to coherently combine all of the propagating energy in the water column is inherent in the use of modal filtering over all of the propagating modes.

The temporal dispersion of the energy propagating in a single mode is always less than or equal to the total temporal dispersion of the channel. The magnitude of the inequality is dependent on the amount of modal coupling in the propagation channel as well as bandwidth of the transmitted signal. In addition, the fading of single-mode amplitude is usually less than joint channel fading. 
Separating energy by mode filtering to independent channels with a reduced delay spread and fading can improve the communication system performance.

The simplest approach to data processing can be selecting the most energetic channel of the mode filter and using it for data decoding. It will be shown below that even such a simplified approach can give good results. The considered approach is making a communication receiver composed of two blocks: the first being a mode-filtering channel-shortening equalizer and the second being an optimal decoder. The mode-filtering preprocessing will decrease the channel delay spread and the level of amplitude fading and increase the SNR. After the preprocessing channel-shortening filter, the channel parameters will have a lower dimension and be better conditioned. As a result of delay-spread shortening, the optimal maximum-likelihood sequence detection (MLSD) can then be applied [18].

There are other approaches to reduce the channel delay spread with a channel-shortening filter: spatial-temporal linear minimum mean-square estimation (MMSE) equalizer and partial-response equalizers [19], passive time-reversal equalizer [20], multicarrier modulation [21], and delay-spread shortening eigenfilter [22]. The passive time-reversal filter shows a very good performance in the underwater communications [20]. The MMSE equalizer is the optimal system, but it is very complicated and needs a long time for adaptation. Partial-response equalizers show good performance for underwater communications. The delay-spread shortening eigenfiltering shows a very promising results for mobile-cellular communications [22]. The last approach is probably the closest radio-frequency (RF) system analog for mode filtering. This paper shows that the mode-filtering processing can be very effective for shallow-water low-frequency communications.

The paper presents the analysis of the data collected during SW06. The objective is to demonstrate on simple examples the feasibility of broadband mode decomposition as a preprocessing method to reduce the effective channel delay spread and to concentrate received signal energy in a small number of independent channels with reduced fading.

\section{The Basics of Acoustic Field Normal-Mode DECOMPOSITION AND MODE FILTERING}

Mode-filtering analysis is based on the normal-mode decomposition of received acoustical fields. We will assume that under shallow-water conditions the propagating acoustical field $p(r, z)$ can be expressed in the normal-mode representation [23], [24]

$$
\begin{aligned}
p(r, z)=\frac{i}{p\left(z_{s}\right) \sqrt{8 \pi r}} & \exp (i \pi / 4) \\
& \times \sum_{m=1}^{\infty} \Psi_{m}\left(z_{s}\right) \Psi(z) \frac{\exp \left(i k_{\mathrm{rm}} r\right)}{\sqrt{k_{\mathrm{rm}}}}
\end{aligned}
$$

where $\Psi(z)$ is a normal-mode function depending only on depth $z, k_{\mathrm{rm}}$ are normal-mode wave numbers, $r$ is the range, and $z_{s}$ is the sound source depth.
The mode shapes can be calculated from the normal-mode differential equation

$$
\frac{d^{2}}{d z^{2}} \Psi_{m}(z)+\left[\frac{\omega^{2}}{c(z)}-k_{\mathrm{rm}}^{2}\right] \Psi_{m}(z)=0
$$

where $c(z)$ is the sound-speed profile, and $\omega=2 \pi f$ is the acoustic frequency.

Signals from the hydrophone array can be represented as

$$
p\left(z_{k}, f\right)=\sum_{m=1}^{M} A_{m}(f) \Psi_{m}\left(z_{k}, f\right)+n\left(z_{k}, f\right) .
$$

In a matrix discrete form, it transforms to

$$
P=\Psi \cdot A+N
$$

where $P=\left\{p_{k}\right\}=\left\{p\left(z_{k}, f\right)\right\}, N=\left\{n_{k}\right\}=\left\{n\left(z_{k}, f\right)\right\}, A=$ $\left\{A_{m}\right\}=\left\{A_{m}(f)\right\}$ are vector columns for sound-pressure observations, acoustical noise, and mode amplitudes correspondingly; $\Psi=\left\{\Psi_{k}^{m}\right\}=\left\{\Psi_{m}\left(z_{k}, f\right)\right\}$ is the matrix of acoustical mode shapes. In the former equation, vector $P$ is data from the hydrophone-array observation. Columns of matrix $\Psi$ are the mode shapes, obtained from the differential-modal equations, where the sound-speed profile is calculated from the temperature and salinity-sensor data. In (4), the vector $P$ and the matrix $\Psi$ are given, and the modal amplitudes $A$ are unknown. It is a standard linear inverse problem with well-known solutions in a sense of different variants of minimum squares based on a different level of a priori information knowledge. These methods have no underwater acoustic specifics and are not described here. The best algorithm minimizes the effect of noise and mode cross talk on the output of the mode filter. Experimental comparison of different methods has shown that total least squares (TLS) and Moore-Penrose PI have the closest and best performance for actual practical problem conditions. The implicit assumption of the least squares solution to a system of equations $P=\Psi \cdot A$ is that all errors are contained in the vector $P$. In contrast, the total least squares solution to this equation assumes that both the vector $P$ and the matrix $\Psi$ may contain errors. The fact that both methods have shown equivalent performance confirmed that the measured mode shapes were close to the actual shape. The PI that minimizes the least square error has the form

$$
\hat{A}_{\mathrm{PI}}=M \cdot P, \quad M=\left(\Psi^{H} \cdot \Psi\right)^{-1} \cdot \Psi^{H}
$$

where $\hat{A}$ is the estimation of mode amplitudes and $M$ is the pseudoinverse matrix. The useful property of this algorithm is that the energy leakage from one mode filter to the output of the filter for another mode (i.e., the modal cross talk or leakage) is very small. The modified pseudoinverse matrix computation is based on singular value decomposition, keeping only relevant singular values. The mode-filtering analysis in this paper will be done by such version of the broadband pseudoinverse method. Other more complicated methods need adaptation for unknown parameters, which makes them less attractive for application. Inverse matrixes are frequency dependent and in a broadband case must be applied for each frequency in the signal spectrum. This broadband approach was considered in many 


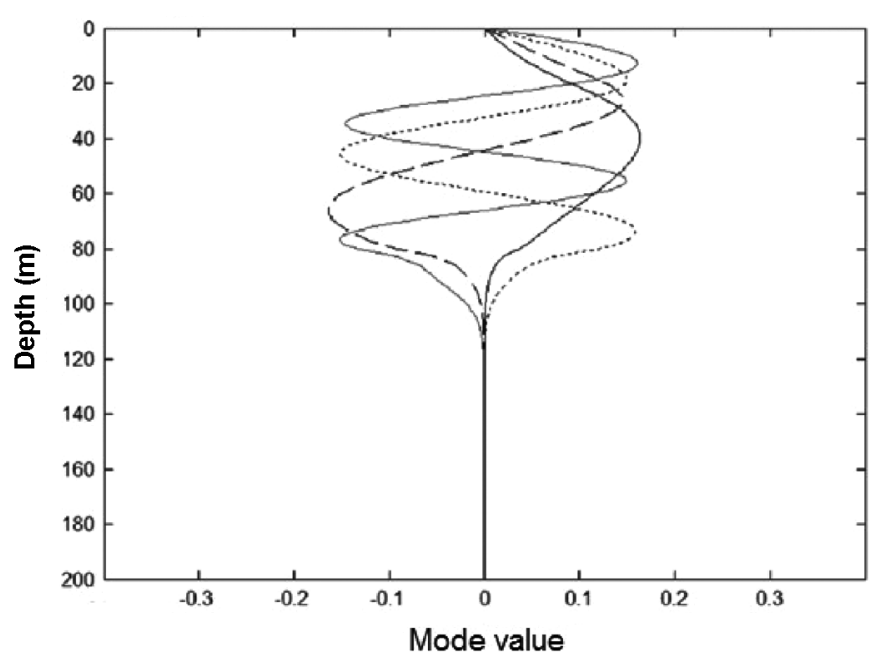

(a)

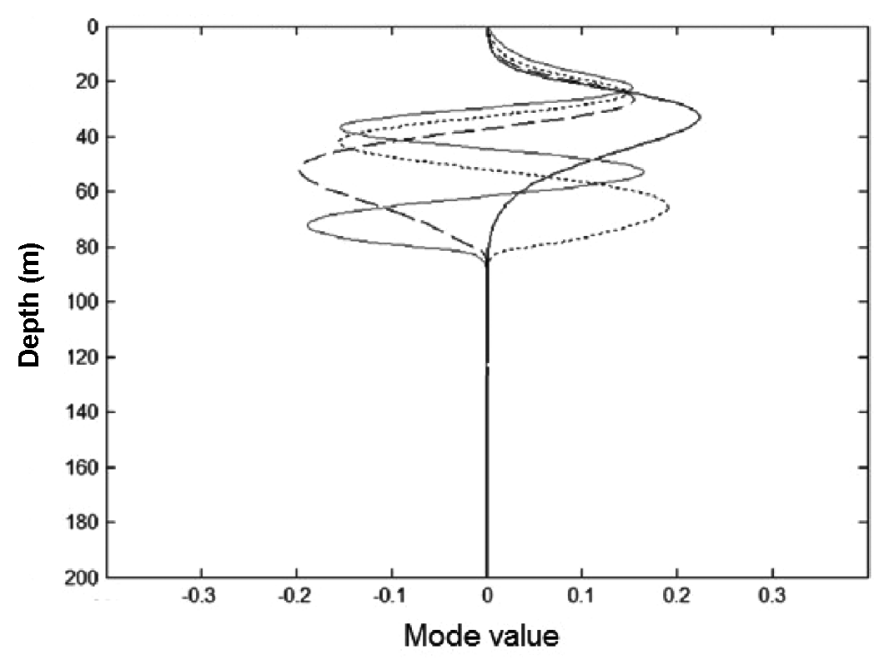

(b)

Fig. 1. Acoustic modes for different frequencies: (a) $f=104 \mathrm{~Hz}$; (b) $f=308 \mathrm{~Hz}$.

papers for a number of different applications: [6], [8], [25], [9], [11], [12]. In these works, the mode coefficients are expected to be constant during the interval of Fourier transformation. The Fourier transform interval determines the frequency resolution of broadband-mode coefficient inversion and must be long enough. The short-time Fourier transform is a standard signal processing technique for examining the characteristics of transient or time-varying signals. The method was applied to time-variable mode filtering [11]. The specifics of mode filtration for underwater acoustics are that mode coefficients are carrying information and therefore rapidly change during a time interval much shorter than during the interval necessary for Fourier transformation. The mode filter transforms continuous streams of samples from hydrophone signals into mode coefficient samples on the output. The broadband mode filter can be built in the frequency domain using the overlap-add method, or in the time domain. The transition to the complex envelopes and another sampling rate can easily be included in the filter structure. An explicit digital signal processing (DSP) algorithm of such mode filter is briefly considered in the Appendix. The frequency-domain approach, described in the Appendix, is a computationally efficient solution for mode filtering. The similar lowpass-equivalent method (baseband mode filtering) was also tested and gave the equivalent result with less computation. The dual time-domain approach suggests estimation of the equivalent multiport mode-filter impulse response with the help of inverse Fourier transformation of frequency-dependent matrix $M(f)$.

All calculations in the following experimental data processing were done by a frequency-domain mode filter based on the overlap-add method. The data from temperature and salinity sensors were averaged over the signal-transmission interval and the result was used for the mode shape calculations for a whole block of received data.

Bottom sound speed, density, and attenuation were taken from [26]. A Kraken algorithm was applied for each frequency in the signal bandwidth to calculate frequency-dependent acoustical modes and their wave numbers. Each $m$-sequence signal has four carrier periods in one data symbol and its bandwidth is $2 \Delta f=0.25 f_{0}$, where $f_{0}$ is the carrier frequency. The frequency-dependent mode functions were calculated for the whole signal frequency bandwidth.

An example of acoustic modes calculated for different frequencies is shown in Fig. 1 for a real sound-speed profile on August 6, 2006.

\section{EXPERIMENT CONDITIONS}

The experimental conditions and techniques are shown in Figs. 2-5. The chart and bathymetry of the experiment are shown in Figs. 2 and 3. It should be noted that the proposed method of communications is sensitive to the bathymetry. It should work perfectly only in shallow water with mildly range-dependent environment between the transmitter and the receiver, where adiabatic mode theory is applicable. The bathymetry shows that in the real experiment the propagation path was across the bottom slope. The positions of the array and the sound source are shown in Fig. 2. The internal wave solitons were generated by tidal waves at the bottom slope and propagated toward the open ocean, so that their fronts were usually along the communications route. A complicated 3-D sound diffraction by the internal wave solitons resulted in strong signal scattering.

The acoustical receiver array is shown in Fig. 4. You can see in Fig. 4 the vertical and horizontal parts of the array. The processed data was collected by a 48-element array called Shark, designed at the Woods Hole Oceanographic Institution (WHOI, Woods Hole, MA [27]). The array system included a 16-channel vertical line array (VLA) and a 32-channel horizontal line array (HLA). This work considers the processing of only the VLA data.

The system was deployed in $78 \mathrm{~m}$ of water, which allowed 13 of the 16 vertical array channels to span the water column from about 77- to 13-m depth. The VLA section was too long for the water depth of $78 \mathrm{~m}$, so the lower three hydrophones were all about $1 \mathrm{~m}$ off the seabed. The hydrophone depths are presented in Table I. 

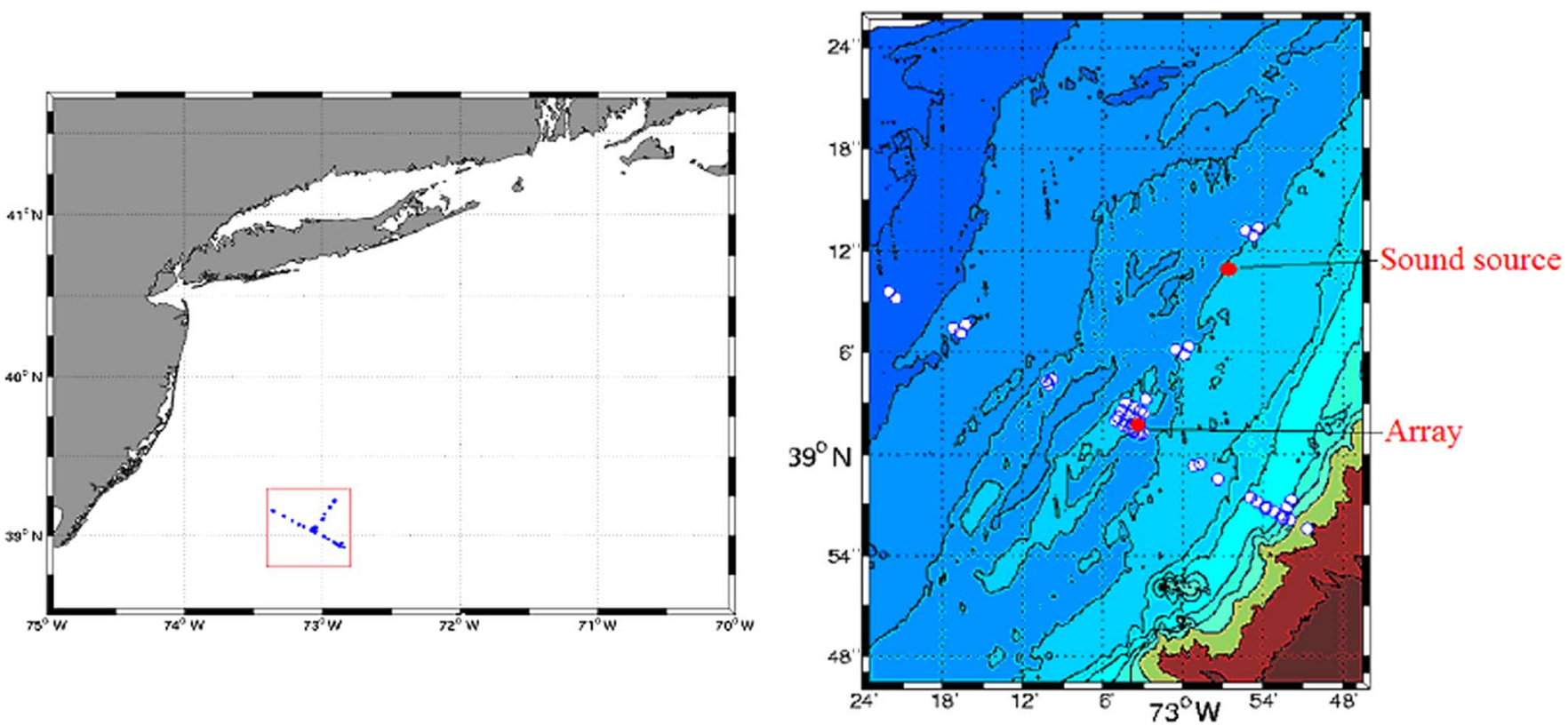

Fig. 2. Chart of the experiment area. The SW06 moorings shown by dots and circles were deployed in a "T" geometry to create an along-shelf path and an across-shelf path. The path along $80-\mathrm{m}$ isobath was investigated in the paper. The top view bathymetry contours are at 40, 50, 60, 70, 80, 90, 100, 120, 150, 250, 500 , and $1000 \mathrm{~m}$.

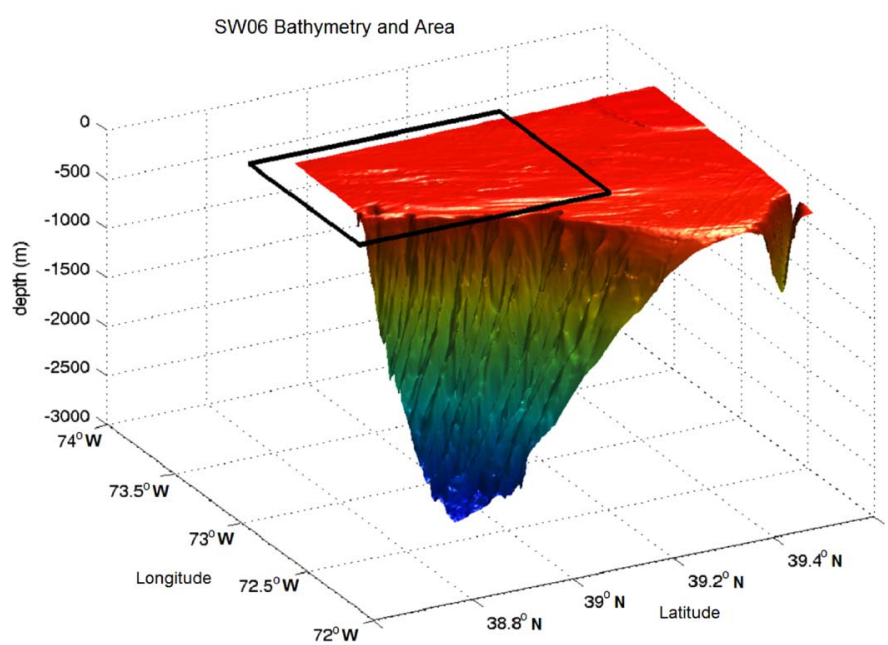

Fig. 3. Experiment bathymetry.

For broadband mode processing, the first 13 hydrophones were used. Three bottom hydrophones from the VLA were approximately at the same depth and only one of them was used for data processing. The Miami Sound Machine (MSM) sound source [28] was moored $20 \mathrm{~m}$ above the sea bottom at a distance of $19.2 \mathrm{~km}$. The sound pressure level (SPL) of the transmitter was limited to approximately $186 \mathrm{~dB}$ re $1 \mu \mathrm{Pa}$ at $1 \mathrm{~m}$, which is typical for the widely used sound sources for RAFOS floats tracking. RAFOS is the word SOFAR (SOund Fixing And Ranging) spelled backwards.

The following binary $180^{\circ}$ phase-shift-keyed (BPSK) signals, emitted from the sound source, have been processed: 34 periods of 63-digit $m$-sequence with the carrier frequency at $101.7253 \mathrm{~Hz}$ and data rate of $25.4 \mathrm{~b} / \mathrm{s} ; 34$ periods of 127 -digit $m$-sequence with the carrier frequency at $203.4505 \mathrm{~Hz}$ and data rate of $50.9 \mathrm{~b} / \mathrm{s}$; and 36 periods of 511-digit $m$-sequence with the carrier frequency at $813.8021 \mathrm{~Hz}$ and data rate of 203.45 $\mathrm{b} / \mathrm{s}$. The analysis result includes data processing of three different days, including times with typical internal wave activity and a case with an abnormally strong internal wave soliton. The sound speed was measured by ten sensors attached to the VLA at the depths: $13,14.8,18.5,22.3,26,33.6,41,56,71$, and 78.3 $\mathrm{m}$. The sound speed was measured every $30 \mathrm{~s}$. The mode processing used an averaged value of the sound-speed profile for the time interval covering the signal length. Bottom parameters used in processing were sound speed, density, and attenuation coefficient.

The MSM sound source is shown in Fig. 5.

The acoustic long baseline navigation system installed on the array included an interrogator at the end of the HLA tail and two transponders from both sides of the HLA. The interrogator and transponders were located in a triangular pattern around the VLA. The distance between transponders was approximately $1000 \mathrm{~m}$. The horizontal distance from interrogator to VLA was approximately $600 \mathrm{~m}$. The interrogator was controlled electronically from the array controller. The local sound-speed profile was taken into account to correct propagation delays and the position of the top hydrophone was calculated by means of least squares fit four times an hour.

\section{Mode-Filtering Performance Estimation}

To check the quality of the mode filtering, the filter output was correlated with the reference $m$-sequence phase-manipulated code and a plot of the complex signal constellation was examined. The correlation processing was used only to estimate the quality of the mode filtering. To recover the transmitted data, the maximum-likelihood (ML) sequence decoder processed the signal as a random BPSK signal. The correlation of mode-filter output with the reference signal shows the delay spread or time scattering of the acoustic energy after the mode filter, as shown 


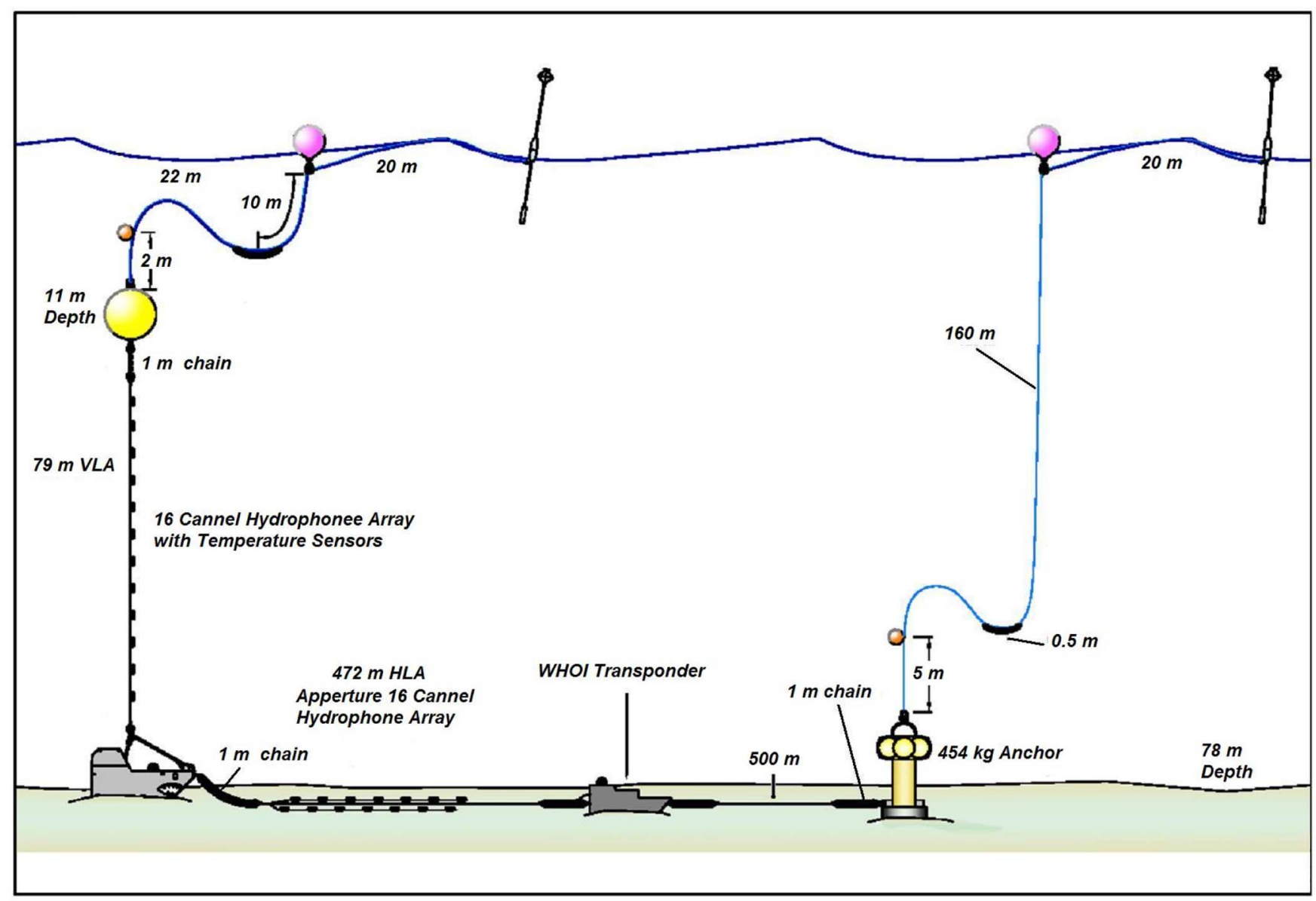

Fig. 4. Receiver array Shark.

in Fig. 6. The results in Fig. 6 were averaged over a single data transmission package. Correlations of the received signal with the reference signal with a shifted carrier frequency show that there was no relevant Doppler frequency shift of the received signals.

The intensity of internal-wave-induced sound-speed fluctuations and the statistics of mode coefficients specifically for SW06 were studied simultaneously with the acoustic communication experiment and results were published in [15] and [29]-[31].

The results for two different cases are presented below, one for August 6, 2006 and another for August 14, 2006. The internal wave activity on August 6, 2006 was a "typical" internal wave random-background field with tidal current-induced internal waves. Such internal wave activity was observed during most of SW06. The internal wave activity on August 14, 2006 was at a much higher level, which led to strong mode coupling and fluctuations of channel parameters. Another "extremely strong" internal wave solitons activity that occurred on August 19, 2006 was presented in [16]. This case was not typical, as such high level of internal activity was observed only once during SW06. The analysis of such different variants is necessary for understanding the limitations of the mode-processing method. The correlation pulses for the aforementioned two examples at different frequencies $(102,203$, and $814 \mathrm{~Hz})$ are presented in Fig. 6 . The maximum energy at the frequency $102 \mathrm{~Hz}$ is concentrated in the first two modes. The first three modes were relevant for communication at the frequency 203 $\mathrm{Hz}$. If single-mode receiver bit error rate (BER) is smaller than 0.1, the mode energy or SNR was considered relevant for communication. In the considered experiment conditions, it means that intensity of insignificant modes was approximately ten times less than the maximum mode intensity. The intensity of the first two modes fluctuates and the position of the most intensive mode was switching between the first and second modes, although the third mode is also relevant.

The acoustic mode spectrum for $814 \mathrm{~Hz}$ is more complicated. More than five acoustic modes were relevant for communication with a long time-spread tail in the impulse response due to mode coupling and mode channel cross talk.

The top array hydrophone displacements during the two different days of the experiment are shown in Fig. 7. The maximum displacement recorded on August 6, 2006 was $T l=\sim 2.5 \mathrm{~m}$. This means that for the first mode with the frequency $814 \mathrm{~Hz}$ during maximum displacement the top half of the array was in antiphase with the bottom half and the array performance degraded dramatically. The frequency limit for such tilt is $f_{\max }=$ $0.5 \mathrm{C} / T l=300 \mathrm{~Hz}$. Tilt compensation in real time is not a practical procedure and was not applied in the presented experiment. The $300-\mathrm{Hz}$ frequency was the highest operating frequency on the Shark VLA that was studied in SW06.

The sparse structure of the array and the limited number of hydrophones are additional problems for high frequencies. However, it will be shown below that, despite degraded per- 


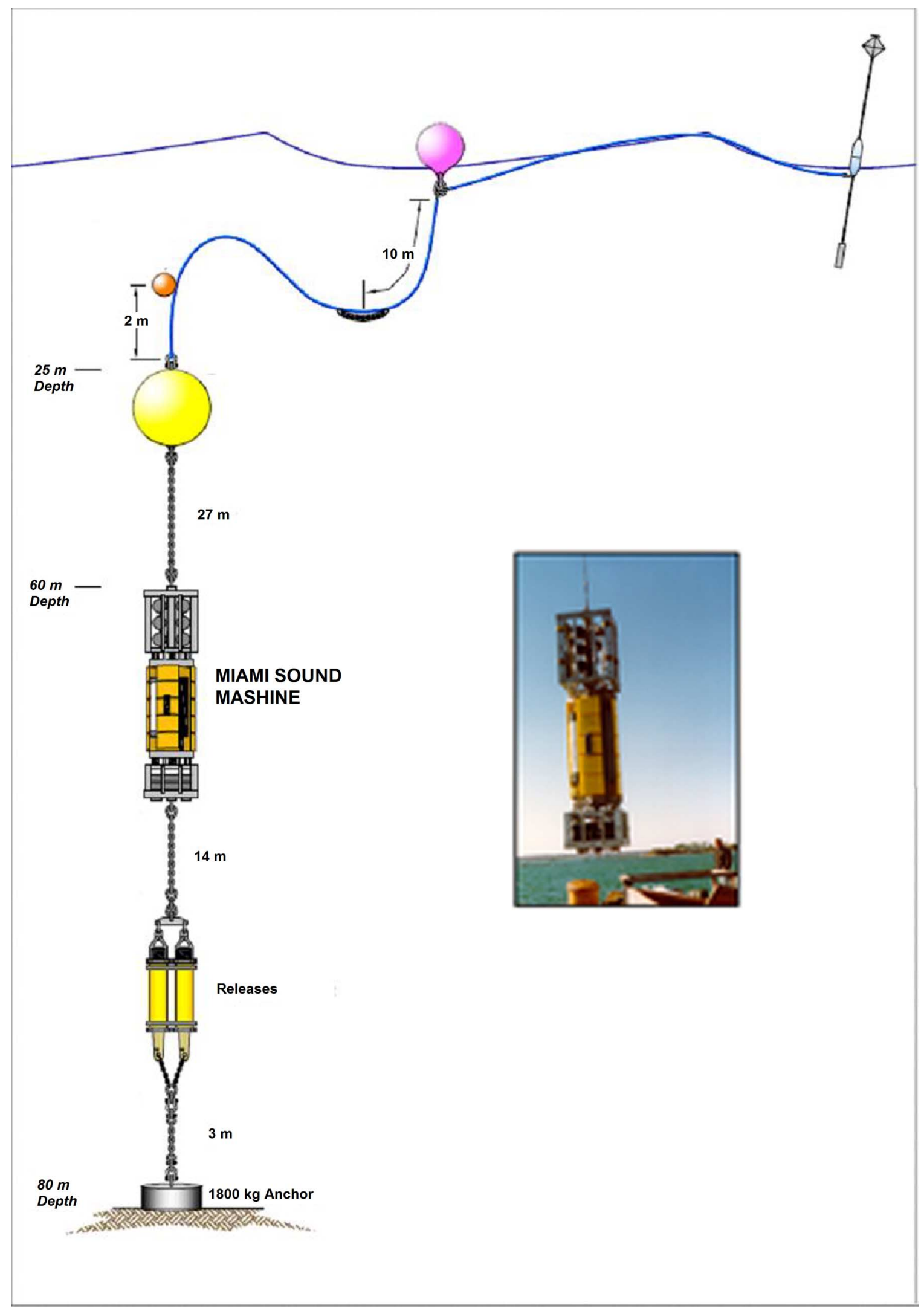

Fig. 5. Acoustic source.

formance, the receiver at $814 \mathrm{~Hz}$ continued operating with a larger, but reasonable error rate.

SNR was continuously monitored during signal reception. The root mean square (rms) level of noise was measured using an interval free from signal just before the beginning of data transmission. The signal-plus-noise rms level was obtained from the record, containing the signal. SNR for each hydrophone was calculated from these two measurements. The same procedure 

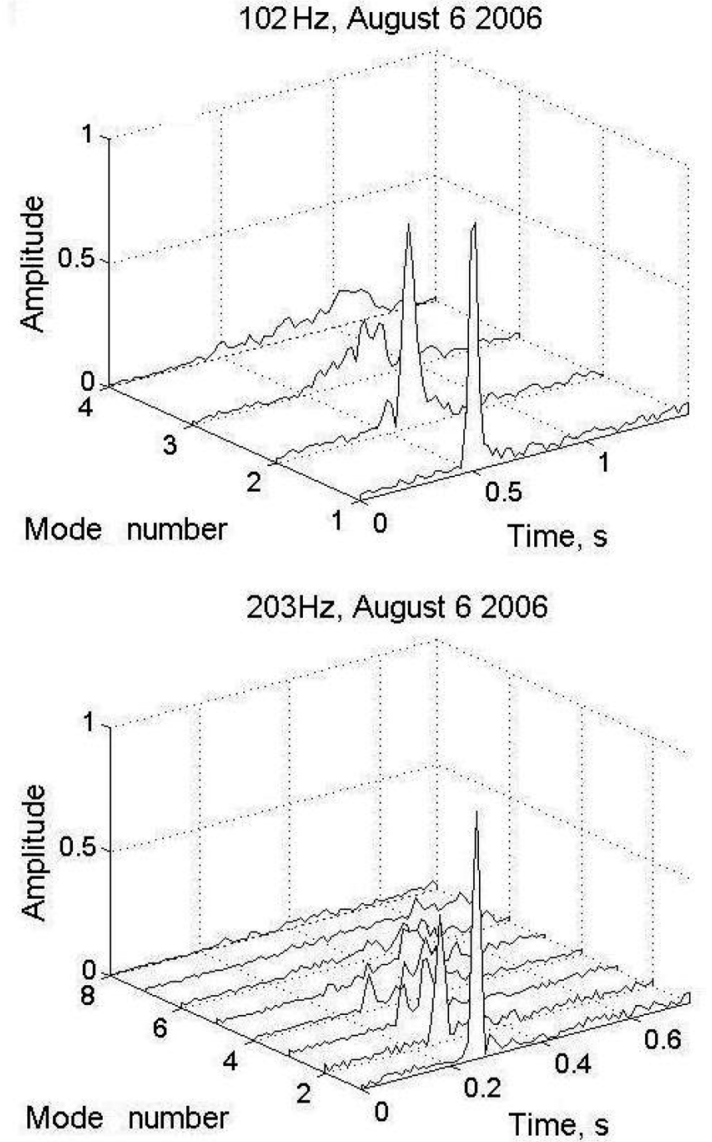

$814 \mathrm{~Hz}$, Aug 62006

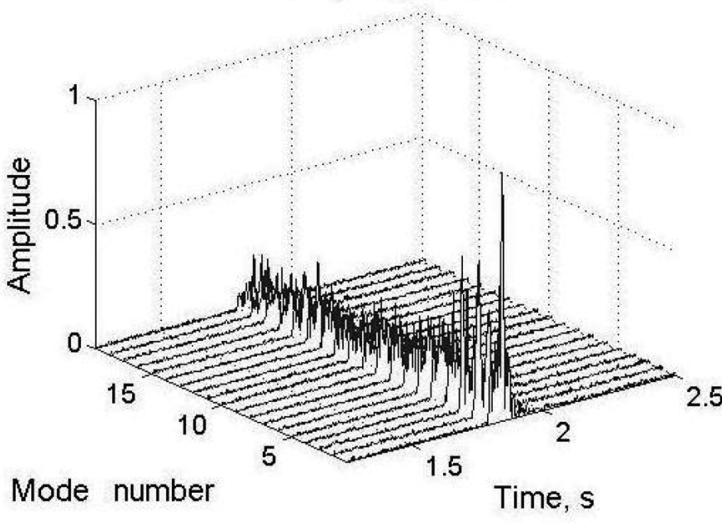

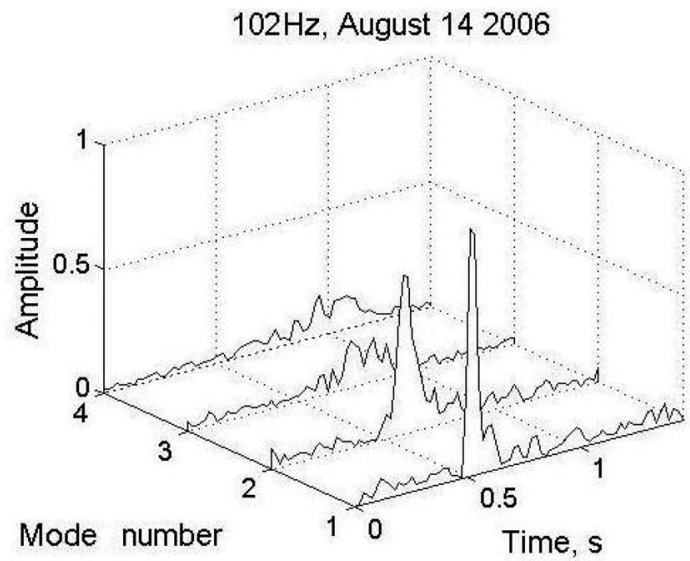

$203 \mathrm{~Hz}$, August 142006

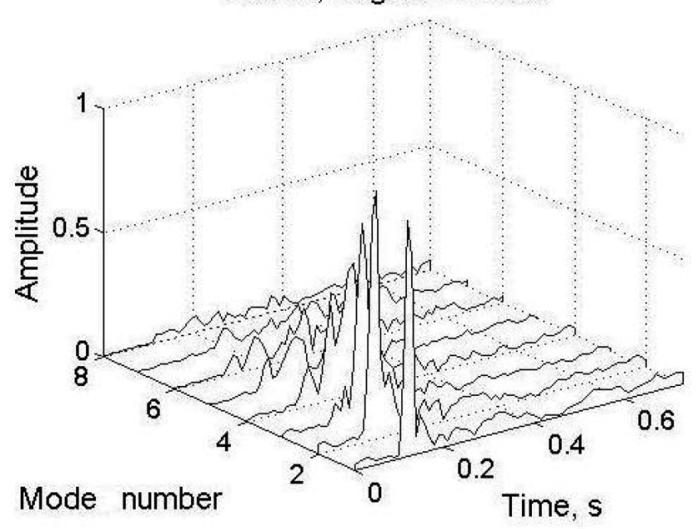

$814 \mathrm{~Hz}$, Aug 142006

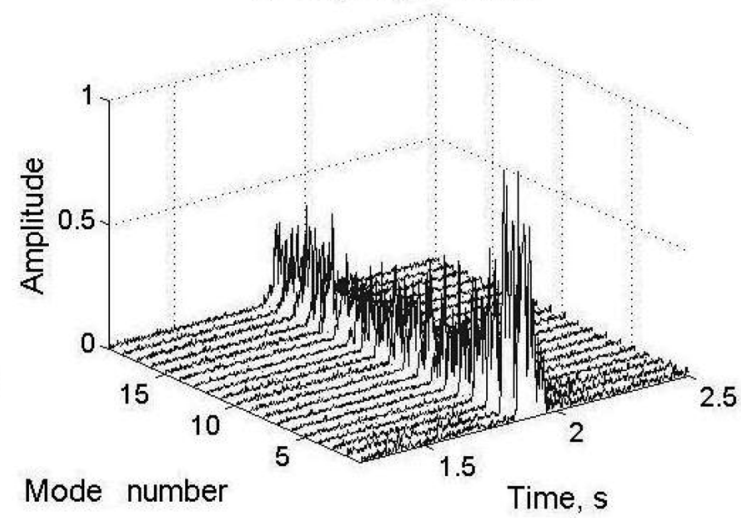

Mode number

Fig. 6. Mode-time correlations.

was used to measure the SNR after mode filtering. The ratio of SNR from the output of the mode filter to the average SNR at the hydrophones will be defined as the mode filter's gain. Signal and noise intensity were depth dependent and time varying. Acoustic noise had a maximum intensity in the middle of an array and decreased intensity near the bottom and the sea surface. As a result, SNR was different at different hydrophones with its mean value varying in time. Typically, the SNR mean value for each hydrophone was below $6 \mathrm{~dB}$ at $203 \mathrm{~Hz}$ and below $9 \mathrm{~dB}$ at $102 \mathrm{~Hz}$. At the maximum output of the mode filter, the SNR level at $203 \mathrm{~Hz}$ increased $9 \mathrm{~dB}$, and increased $10 \mathrm{~dB}$ at $102 \mathrm{~Hz}$. Despite the fact that the mode filter selected only one mode containing a fraction of the total acoustic signal energy, the mode filter's gain was still near the optimal value. Another advantage of the mode filtering was much less channel fading.

The conclusion states that the mode filtering decreased the time spread and fading of the received signal and increased the SNR. The considered type of VLA can be used for very effective mode filtering at a frequency of approximately $200 \mathrm{~Hz}$.

\section{Data Communication Results}

$M$-sequences can be considered independent binary random data with phase shift keying at $180^{\circ}$. Each symbol of such data 
TABLE I

ARRAY HYDROPHONE DEPTHS

\begin{tabular}{|c|c|c|c|c|c|c|c|c|c|c|c|c|c|c|}
\hline Hydrophone number & 1 & 2 & 3 & 4 & 5 & 6 & 7 & 8 & 9 & 10 & 11 & 12 & 13 & 14 \\
\hline Depth $(\mathrm{m})$ & 13.50 & 17.25 & 21.00 & 24.75 & 28.50 & 32.25 & 36.00 & 39.75 & 43.50 & 47.25 & 54.75 & 62.25 & 69.75 & 77.25 \\
\hline
\end{tabular}
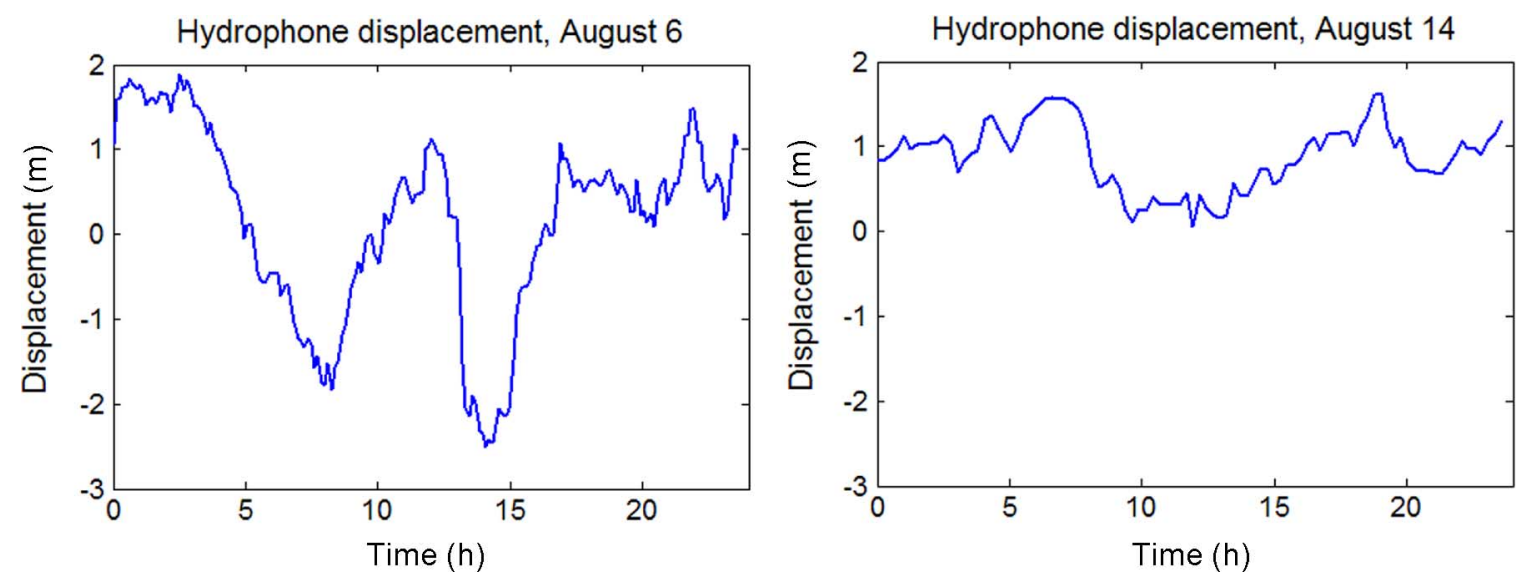

Fig. 7. Horizontal top hydrophone displacement in the direction to the sound source.

transmission carries $1 \mathrm{~b}$ of information. No $m$-sequence properties or structures were used for data processing during signal receptions; no pilot or training signals were used. The good shape of the signal correlations, small time spread, low-fading level, and high SNR at the output of the mode filter suggest that good results can be expected from data recovery by any kind of simple decoder. The important advantage of the mode-filtering approach is that channel parameters' complexity can be reduced to the level of usual RF channels and sophisticated optimal decoders can be used. In this research, a state-limited ML sequence decoder [18] with a short joint finite impulse response (FIR) channel parameters estimator was applied. A joint channel estimation and data recovery algorithm in the form of presurvivor processing [32] was identical to [16], and is briefly described below. Specifically, the method realized the joint minimization of metrics $d_{l}=\left|y_{l}-\sum_{m=0}^{N} \hat{h}_{l m} k_{l-m}\right|$ by means of Veterbi trellis algorithm and stochastic approximation, changing channel estimation on each step in the direction opposite to gradient of the metrics.

The discrete form of the channel at the output of the mode filter is

$$
y_{l}=\sum_{j=1}^{N} h_{l j} k_{l-j}+n_{l}
$$

where $n_{l}$ is the white Gaussian noise and $h_{j}(t)$ or $h_{j l}$ are the low-frequency equivalents of time-variable channel pulse response; $k_{j}= \pm 1$ is the modulating parameter; and $x(t)$ is the sequence of the data signals $s(t)$ modulated by $k_{j}= \pm 1$.

The simplified equations for the ML metrics $w_{l}$ are

$$
\begin{aligned}
w_{l} & =w_{l-1}-\left|d_{l}\right|^{2} \\
\hat{h}_{l m} & =\hat{h}_{l-1, m}-a d_{l} k_{l-m} \\
d_{l} & =y_{l}-\sum_{m=0}^{N} \hat{h}_{l m} k_{l-m}
\end{aligned}
$$

where $\hat{h}_{l m}$ are least mean squares (LMS) estimates of the channel impulse response obtained in accordance with (8) and (9). The coefficient $a=0.001$ is the stepsize of the estimation algorithm. The stepsize value is responsible for the compromise between errors introduced by observation noise and those resulting from temporal channel fluctuations. The algorithm operates by iterations (7)-(9) for each sample with the rate of two samples per symbol. The algorithm calculates trellis metrics of the standard Viterbi trellis in accordance with (7) and includes an LMS adaptive channel estimator (8) for each sequence of data symbols (for each branch of the trellis). The codes for data recovery and channel parameter estimation have much in common, and the entire modified MLSD program is relatively simple.

Transmissions during two different experimental days, August 6, 2006 and August 14, 2006, with different internal wave activity were analyzed, and results are presented in Figs. 8 and 9. The first row in Fig. 8 shows internal-wave-induced soundspeed fluctuations as a function of the time of a day. Periodically (typically twice a day), linear random internal wave fluctuations were transformed into strong nonlinear solitons. The second and third rows in Fig. 8 show the mode-amplitude fluctuations. These fluctuations are more active for the $203-\mathrm{Hz}$ signal with its maximum intensity periodically switching from the first mode to the second mode many times a day. At $102 \mathrm{~Hz}$, signal fluctuations are much slower. Although the maximum of the mode spectrum is slowly drifting between the second and first modes, the first mode remains very strong almost all the time. The simplest variant of the receiver was considered where it automatically decoded the signal from the most reliable mode channel. More complicated approaches use optimally weighted sums of time-synchronized signals from different mode channels.

Constraint lengths for the decoder trellis were limited correspondingly by three pulse intervals for August 6, 2006 and by five pulse intervals for August 14, 2006 data processing. A short starting piece of data with the adaptation transient process was not accounted for error calculations. 

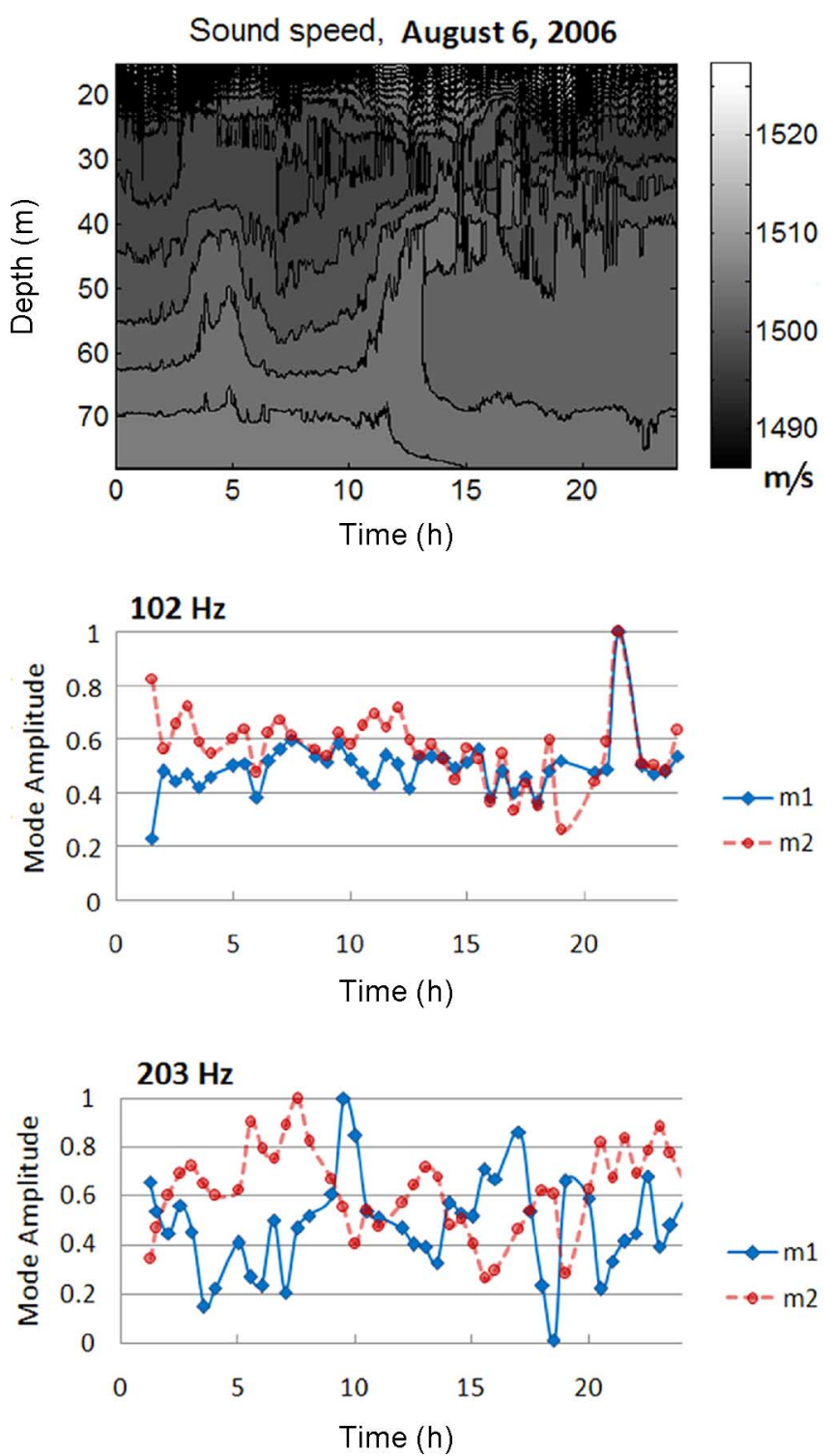

Fig. 8. Sound speed and mode amplitude variability during August 6 and 14, 2006
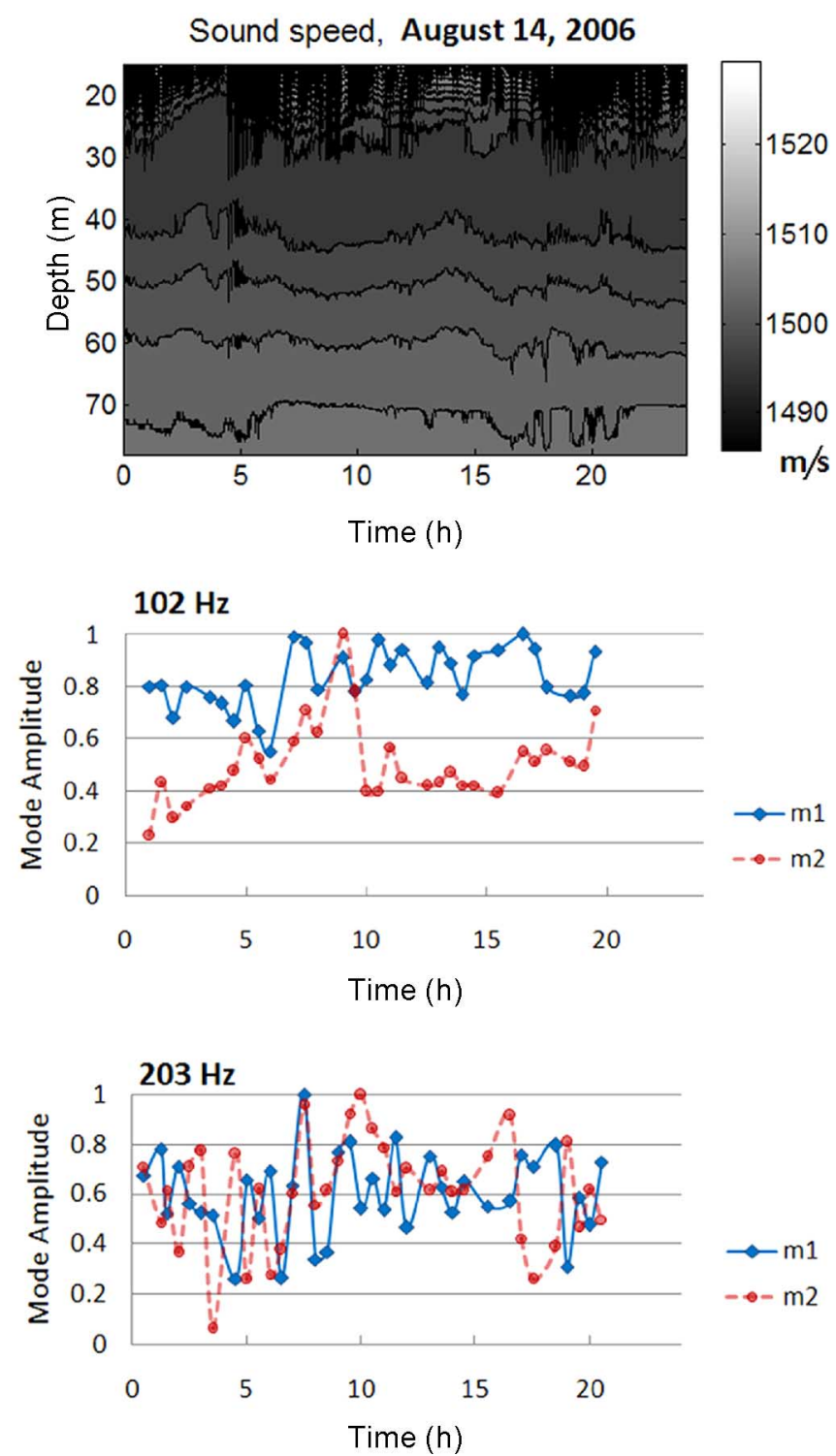

The BERs for the two days are shown in Fig. 9. The graph indicates the best BER between all received modes, and only this BER is shown. The acoustic mode channels with the best error rate have different markers: mode 1 -circle; mode 2-square; mode 3-diamond. The BER level $10^{-4}$ corresponds to zero. At $102 \mathrm{~Hz}$, the BER was almost always zero for the first mode. The communication channel at $203 \mathrm{~Hz}$ was much more sensitive to the strong soliton waves, when its performance degraded to the BER of $10^{-2}$. The data from the array positioning system does not show cable inclination, which can be the only reason for such degradation. The BER increases because of a decrease in SNR, due to noise rising and mode coupling. The former effect was more important among these two. The nature of that noise is more likely connected to the close research vessel activity following the position of internal waves.

To estimate receiver BER sensitivity to SNR, a numerical simulation was used with a recorded digital received signal after mode filtering was done. The BER for different SNR in a given modal channel was estimated by adding white computer-generated noise to the actual received signal. The results for August 6,
2006 for the second mode and the 203-Hz frequency are shown in Fig. 10. The SNR was varied from 0 to $15 \mathrm{~dB}$; for $15 \mathrm{~dB}$, BER $=0$ is not shown in the graph.

The three problems of cross talk due to array displacement, mode coupling, and noise became much more serious at the higher frequency of $814 \mathrm{~Hz}$ and caused a high BER. The BERs for the example in Fig. 6 are as follows:

- August 6, 2006: mode 1, BER $=0.05$; mode 2, BER = 0.35 ; mode $3, \mathrm{BER}=0.055$; mode 4 , $\mathrm{BER}=0.3$

- August 14, 2006: mode 1, BER $=0.04$; mode 2, BER $=0.34$, mode $3, \mathrm{BER}=0.07$; mode $4, \mathrm{BER}=0.02$.

Results for $814 \mathrm{~Hz}$ were not as good as for 102 and $203 \mathrm{~Hz}$, although in those cases, all the energy was concentrated in the first few modes with a small time spread. The processing for higher frequencies needs a much denser array and correction for array tilt.

\section{CONCLUSION}

The broadband mode filtering allows one to decompose the received signal into a number of independent signals with a re- 

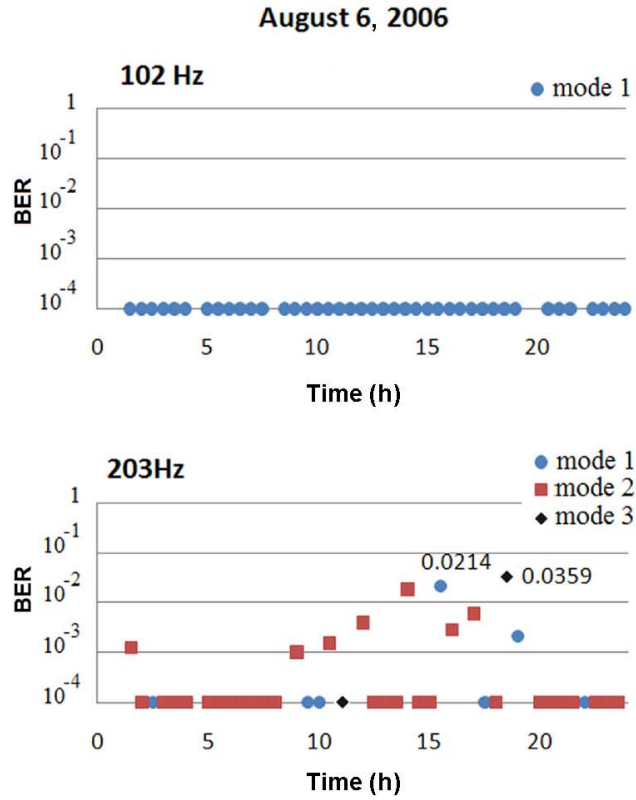

Fig. 9. Bit error rate.

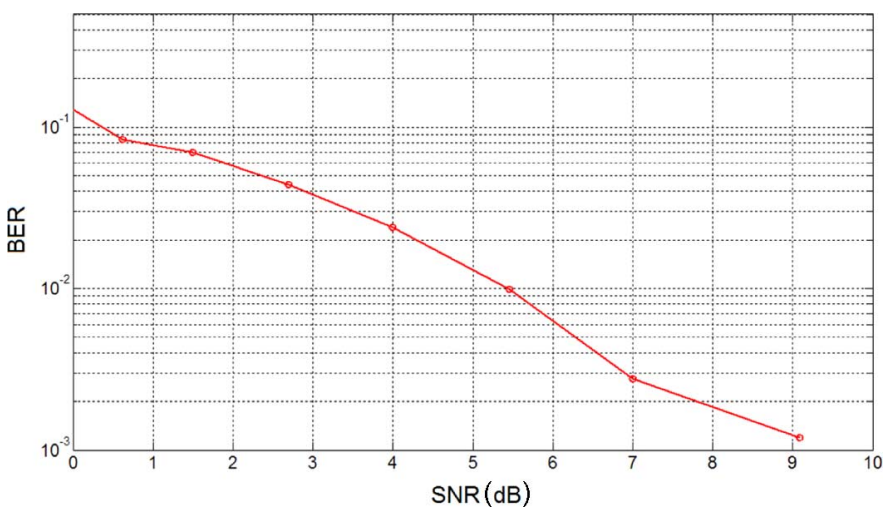

Fig. 10. BER versus SNR in a selected modal channel.

duced channel delay spread. The sharp correlations of signals from the output of mode filters and large SNR show that even a simple demodulator can achieve a high-quality reception. The mode-filtering method performed very well at 102 and $203 \mathrm{~Hz}$ and has satisfactory performance at $814 \mathrm{~Hz}$. The data processing shows that even in a very complicated environment with strong internal wave solitons, the acoustical energy is concentrated in a small number of the first acoustical modes. A receiver can estimate the mode-time intensity distribution and use a signal from a more intensive mode (or a few of them) for demodulation. A very high-quality data transmission can be achieved for a range of approximately $19.2 \mathrm{~km}$ at the frequencies 102 and $203 \mathrm{~Hz}$. Some additional properties of mode filtering for communication, such as constellations of complex envelopes at the output of the mode filters for different modes, were discussed in [16]. The comparison of BER for single hydrophone receiver and for the mode-filtering output is discussed in [17] and is not considered in this paper.
August 14, 2006
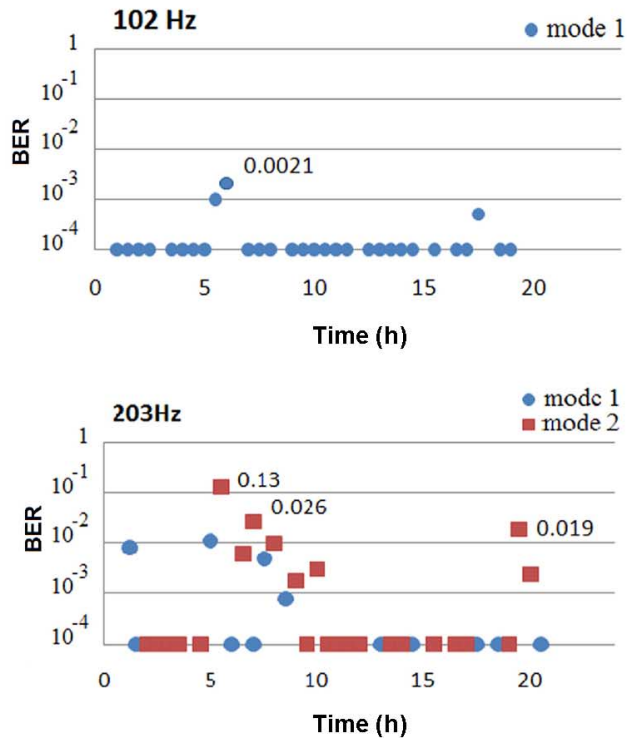

\section{APPENDIX \\ BROADBAND MODE-FILTER DSP IMPLEMENTATION IN FREQUENCY DOMAIN}

The broadband mode filter transforms acoustical array input signals into time-variable mode coefficients at its output. The bandwidth and sampling rate of the input and output signals do not change. The filter works in real time with a small delay, because of the filter time transient response. The filter can be implemented in the frequency domain as a baseband or passband linear system or in the time domain as an array of FIR filters. The former approach is easy for data processing and the second is better for fast DSP implementation.

Let us first consider a frequency-domain passband mode filter based on Moore-Penrose pseudoinverse. The input signal for each hydrophone was sampled and divided into sections $S^{(l)}=$ $\left\{S_{1} ; S_{2} ; \ldots S_{k}\right\}^{T}$, where the superscript $l$ will denote section number in later sections. Each row $S_{j}=\left\{s_{j, 1}, s_{j, 2}, \ldots s_{j, n}\right\}$ of the matrix $S^{(l)}$ is the sequence of passband signal samples from the hydrophone number $j ; s_{j, i}$ is the $i$-sample from $j$-hydrophone. The number of hydrophones is $k$.

Each section for each hydrophone was zero-padded and then Fourier-transformed at 64 frequencies: $\tilde{S}_{j}=$ $\left\{s_{j, 1}, s_{j, 2}, \ldots s_{j \cdot n}, 0,0, \ldots 0\right\} ; X_{j}=\operatorname{FFT}\left(\tilde{S}_{j}\right), X=$ $\left\{X_{1} ; X_{2} ; \ldots X_{k}\right\}^{T}$, where FFT() is the fast Fourier transform; each row $X_{j}$ from the matrix $X$ is the spectrum of zero-padded signal from hydrophone number $j$. The length of each vector row $\tilde{S}_{j}$ and $X_{j}$ is $n_{p}$. The column $X^{i}(f)=\left\{x_{1, i}, x_{2, i}, \ldots x_{k}\right\}$ from matrix $X$ is the sound pressure with the frequency $f$ distributed over $k$ hydrophones. The PI is applied to that column vector at each frequency

$$
\begin{aligned}
& V^{i}(f)=M(f) \cdot X^{i}(f) \\
& M(f)=\left(\Psi^{H}(f) \cdot \Psi(f)\right)^{-1} \cdot \Psi^{H}(f)
\end{aligned}
$$


and output matrix for the processing section $(l)$ stacks from columns $V^{i}, V^{(l)}=\left\{V^{1}, V^{2}, \ldots V^{\left(n_{p}\right)}\right\}$. The samples of mode complex envelope $Z_{m}$ for the mode number $m$ from a processed section are obtained after inverse fast Fourier transformation of each row of matrix $V^{(l)} Z_{m}=\operatorname{IFFT}\left(V_{m}\right)$, where matrix $Z^{(l)}=\left\{Z_{1} ; Z_{2} ; \ldots Z_{k}\right\}^{T}$.

The final operation is combining segments $Z_{m}^{(l)}$ for each mode channel into the long series of output signal samples using the overlap-add method. For $l=0: y_{m, j}=z_{m, j}^{(0)}, 1<j<n$, for $l>0$.

Then

$$
y_{m, n * l+j}= \begin{cases}z_{m, j}^{(l)}+z_{m, j+n}^{(l-1)}, & 1<j<\left(n-n_{p}\right) \\ z_{m, j}^{(l)}, & \left(n-n_{p}\right)<j<n .\end{cases}
$$

The second row in the last equation is the overlap part of the signal segments.

\section{ACKNOWLEDGMENT}

The authors would like to thank Dr. J. F. Lynch and A. Newhall for SW06 experimental data. They would also like to thank Dr. T. Duda, Dr. J. Collis, and Dr. Y. T. Lin for the help in data processing; K. Von Der Heydt for the SHARK acoustic array design; A. A. Shmelev for hydrophones and acoustic navigation data processing; and Dr. H. DeFerrari for the MSM underwater acoustic sound source.

\section{REFERENCES}

[1] R. H. Ferris, "Comparison of measured and calculated normal-mode amplitude functions for acoustic waves in shallow water," J. Acoust. Soc. Amer., vol. 52, no. 3, pp. 981-988, 1972.

[2] F. Ingenito, "Measurements of mode attenuation coefficients in shallow water," J. Acoust. Soc. Amer., vol. 53, no. 3, pp. 858-863, 1973.

[3] C. T. Tindle, K. M. Guthrie, G. E. J. Bold, M. D. Johns, K. O. Dixon, and T. G. Birdsall, "Measurements of the frequency dependence of normal modes," J. Acoust. Soc. Amer., vol. 64, no. 4, pp. 1178-1185, 1978.

[4] T. C. Yang, "A method of range and depth estimation by modal decomposition," J. Acoust. Soc. Amer., vol. 82, no. 5, pp. 1736-1745, 1987.

[5] J. R. Buck, J. C. Preisig, and K. E. Wage, "A unified framework for mode filtering and the maximum a posteriori mode filter," J. Acoust. Soc. Amer., vol. 103, pp. 1813-1824, 1998.

[6] T. C. Yang, "Broadband source localization and estimation," J. Acoust. Soc. Amer., vol. 93, no. 4, pp. 1797-1806, 1993.

[7] H.-Y. Chen and I.-T. Lu, "Localization of broadband source using a matched-mode procedure in the time-frequency domain," IEEE $J$. Ocean. Eng., vol. 19, no. 2, pp. 166-174, Apr. 1994.

[8] C.-S. Chiu, C. W. Miller, and J. F. Lynch, "Optimal modal beamforming of bandpass signals using an undersized sparse vertical hydrophone array: Theory and a shallow-water experiment," IEEE J. Ocean. Eng., vol. 22, no. 3, pp. 522-533, Jul. 1997.

[9] K. D. Heaney and W. A. Kuperman, "Very low-range source localization with a small vertical array," J. Acoust. Soc. Amer., vol. 104, no. 4, pp. 2149-2159, 1998.

[10] R. H. Headrick, J. F. Lynch, J. N. Kemp, A. E. Newhall, K. Von Der Heydt, J. Apel, M. Badey, C.-S. Chiu, S. Finette, M. Orr, B. Pasewark, A. Turdot, S. Wolf, and D. Tielbuerger, "Acoustic normal mode fluctuation statistics in 1995 SWARM internal wave scattering experiment," J. Acoust. Soc. Amer., vol. 107, pp. 201-220, 2000.

[11] K. E. Wage, A. B. Baggeroer, and J. C. Preisig, "Modal analysis of broadband acoustic receptions at 3515-km range in North Pacific using short-time Fourier techniques," J. Acoust. Soc. Amer., vol. 113, pp. 801-817, 2003.

[12] K. E. Wage, M. A. Dzieciuch, P. F. Worcester, B. M. Howe, and J. A. Mercer, "Mode coherence at megameter ranges in North Pacific ocean," J. Acoust. Soc. Amer., vol. 117, pp. 1565-1581, 2005.
[13] T. F. Duda and J. M. Collis, "Acoustic field coherence in four-dimensionally variable shallow water environments: Estimation using co-located horizontal and vertical line arrays," in Proc. 2nd Int. Conf. Underwater Acoust. Meas. Technol. Results, Crete, Greece, 2007.

[14] Yu. A. Chepurin, "Experiments on underwater acoustic tomography," Acoust. Phys., vol. 53, no. 3, pp. 393-416, 2007.

[15] J. M. Collis, T. F. Duda, J. F. Lynch, and H. A. DeFerrari, "Observed limiting cases of horizontal field coherence and array performance in a time-varying internal wave-field," J. Acoust. Soc. Amer., vol. 124, pp. EL97-EL103, 2008.

[16] A. K. Morozov, J. C. Preisig, and J. C. Papp, "Modal processing for acoustic communications in shallow water experiment," J. Acoust. Soc. Amer., vol. 124, no. 3, pp. EL177-EL181, 2008.

[17] A. K. Morozov, J. C. Preisig, and J. C. Papp, "Investigation of modal processing for low frequency acoustic communications in shallow water," in Proc. 155th Meeting Acoust. Soc. Amer., Paris, France, Jun. 29-Jul. 4 2008, vol. 4, no. 1, pp. 070005-070005-16 [Online]. Available: http://dx.doi.org/10.1121/1.3068504

[18] G. D. Forney, "The Viterbi algorithm," Proc. IEEE, vol. 61, no. 3, pp. 268-278, Mar. 1973

[19] S. Roy, T. M. Duman, V. McDonald, and J. G. Proakis, "High-rate communication for underwater acoustic channels using multiple transmitters and space-time coding: Receiver structures and experimental results," IEEE J. Ocean. Eng., vol. 32, no. 3, pp. 663-688, Jul. 2007.

[20] W. A. Kuperman, W. S. Hodgkiss, H. C. Song, T. Akal, C. Ferla, and D. Jackson, "Phase conjugation in the ocean: Experimental demonstration of an acoustic time-reversal mirror," J. Acoust. Soc. Amer., vol. 102, no. 1, pp. 25-40, 1998.

[21] A. K. Morozov and J. C. Preisig, "Underwater acoustic communications with multi-carrier modulation," in Proc. OCEANS Conf., 2006, DOI: 10.1109/OCEANS.2006.307117.

[22] S. D. Gray, J. C. Preisig, and D. Brady, "Multiuser detection in a horizontal underwater acoustic channel using array observations," IEEE Trans. Signal Process., vol. 45, no. 1, pp. 148-160, Jan. 1997.

[23] F. B. Jensen, W. A. Kuperman, M. B. Porter, and H. Schmidt, Computational Ocean Acoustics. New York: Springer-Verlag, 2000, p. 578 .

[24] L. M. Brekhovskikh and Yu. P. Lusanov, Fundamentals of Ocean Acoustics, 2nd ed. Berlin, Germany: Springer-Verlag, 1991, p. 296.

[25] P. Sutton, W. Morawitz, B. Cornuelle, G. Masters, and P. Worcester, "Incorporation of acoustic normal mode data into tomographic inversions in the Greenland sea," J. Geophys. Res., vol. 99, no. C6, pp. 12 487-12 502, 1994.

[26] Y.-M. Jiang, N. R. Chapman, and M. Badiey, "Quantifying the uncertainty of geoacoustic parameter estimates for the New Jersey shelf by inverting air gun data," J. Acoust. Soc. Amer., vol. 121, no. 4, pp. 1879-1894, 2007.

[27] A. E. Newhall, T. F. Duda, K. Von der Heydt, J. D. Irish, J. N. Kemp, S. A. Lerner, S. P. Liberatore, Y. T. Lin, J. F. Lynch, A. R. Maffei, A. K. Morozov, A. A. Shmelev, C. J. Sellers, and W. Witzell, "Acoustic and oceanographic observations and configuration information for the WHOI moorings From the SW06 experiment," Appl. Ocean Phys. Eng. (AOP\&E), Woods Hole, MA, WHOI Tech. Rep., 2007.

[28] H. B. Nguyen, H. A. DeFerrari, H. A., and N. J. A. Williams, "A general purpose autonomous transmitter and receiver system for underwater acoustic experiments," IEEE J. Ocean. Eng., vol. 21, no. 1, pp. 85-93, Jan. 1996.

[29] J. F. Lynch, Y.-T. Lin, T. F. Duda, and A. E. Newhall, "Acoustic ducting, reflection, refraction, and dispersion by curved nonlinear internal waves in shallow water," IEEE J. Ocean. Eng., vol. 35, no. 1, pp. 12-27, Jan. 2010.

[30] J. Luo, M. Badiey, E. A. Karjadi, B. Katsnelson, A. Tskhoidze, J. F. Lynch, and J. N. Moum, "Observation of sound focusing and defocusing due to propagating nonlinear internal waves," J. Acoust. Soc. Amer., vol. 124, no. 3, pt. 2, pp. EL66-EL72, 2008.

[31] H. A. DeFerrari, J. F. Lynch, and A. E. Newhall, "Temporal coherence of mode arrivals," J. Acoust. Soc. Amer., vol. 124, no. 3, pt. 2, pp. EL104-EL109, 2008.

[32] R. Raheli, A. Polydoros, and C. K. Tzou, "Per-survivor processing: A general approach to MLSE in uncertain environment," IEEE Trans. Commun., vol. 43, no. 234, pp. 354-364, Feb./Mar./Apr. 1995. 


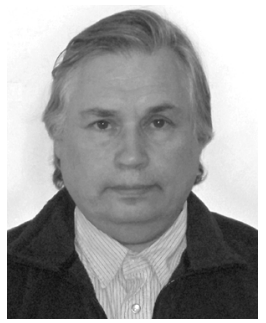

Andrey K. Morozov was born in Moscow, Russia. He received the M.S. degree in radio-physics from the Moscow Power Engineering Institute (Technical University), Moscow, Russia, in 1975, the M.S. degree in applied mathematics from the Moscow State University, Moscow, Russia, in 1984, and the Ph.D. degree from the Moscow Power Engineering Institute, Moscow, Russia, in 1981.

In 1975, he was a Junior Research Scientist at the Moscow Power Engineering Institute, and then became a Senior Scientist and a Deputy Head of the Radio-Systems Department. From 1987 to 1998, he was with the P.P. Shirshov Institute of Oceanology, Russian Academy of Sciences, Moscow, Russia, as a Senior Scientist and then as a Lead Scientist. From 1998 to 1999, he was a Visiting Professor at the Research Institute for Applied Mechanics, Kyushu University, Fukuoka, Japan. He joined Teledyne Webb Research, Falmouth, MA, in 1999 as a Chief Scientist. Since 2001, he has been with Woods Hole Oceanographic Institution, Woods Hole, MA. He has extensive research experience in the field of communications, signal processing, and underwater acoustics.

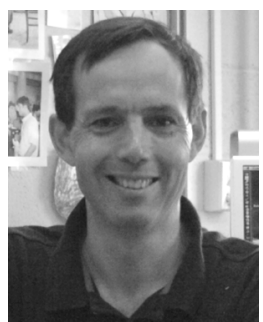

James C. Preisig received the B.S. degree in electrical engineering from the United States Coast Guard Academy, New London, CT, in 1980, the S.M. and E.E. degrees in electrical engineering from the Massachusetts Institute of Technology, Cambridge, in 1988, and the Ph.D. degree in electrical and ocean engineering from the Massachusetts Institute of Technology (MIT)/Woods Hole Oceanographic Institution (WHOI) Joint Program in Oceanography and Oceanographic Engineering, Cambridge/Woods Hole, MA, in 1992.
He was a Postdoctoral Investigator at WHOI from 1992 to 1994 and a Visiting Assistant Professor at Northeastern University from 1994 to 1997. Since July 1997, he has been on the scientific staff of the Department of Applied Ocean Physics and Engineering at WHOI and is currently an Associate Scientist with Tenure. His research interests are in the areas of adaptive signal processing, system identification, underwater acoustic propagation modeling, underwater acoustic communications, and numerical optimization.

Dr. Preisig is the recipient of the 1999 Office of Naval Research (ONR) Ocean Acoustics Young Faculty Award and is a member of the Acoustical Society of America's Underwater Acoustics and Signal Processing Technical Committees. $\mathrm{He}$ is also an Associate Editor of the IEEE JOURNAL OF OCEANIC ENGINEERING and served as a member of the IEEE Sensor Array and Multichannel Signal Processing Technical Committee from 1998 to 2004.

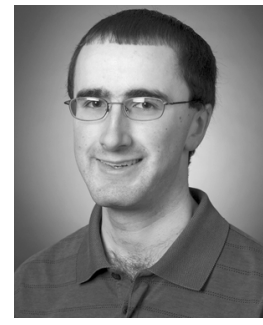

Joseph C. Papp received the B.S. degree in electrical and computer engineering from Worcester Polytechnic Institute, Worcester, MA, in 2006 and the S.M. degree in electrical engineering and computer science from the Massachusetts Institute of Technology (MIT)/Woods Hole Oceanographic Institution (WHOI) Joint Program in Oceanography and Oceanographic Engineering, Cambridge/Woods Hole, MA, in 2009.

In 2006, he worked for MIT Lincoln Laboratory's Ranges and Test Beds group in Lexington, MA. Currently, he is an Associate Staff Member for the same group at MIT Lincoln Laboratory. 